Research Article

\title{
Gram-Scale Synthesis of Pt-Cu Nanowires with Enhanced Electrocatalytic Activity towards Methanol Oxidation Reaction
}

\author{
Ning Sui, ${ }^{1}$ Hongxu Gao, ${ }^{1}$ Yukai Wang, ${ }^{1}$ Jiali Li, ${ }^{1}$ Shiyu Qu, ${ }^{1}$ Qiang Bai, ${ }^{1}$ Hailian Xiao, \\ Jing Sui, ${ }^{1}$ Manhong Liu $\oplus^{1},{ }^{1}$ and William W. Yu ${ }^{1,2}$ \\ ${ }^{1}$ College of Materials Science and Engineering, Qingdao University of Science and Technology, Qingdao 266042, China \\ ${ }^{2}$ Department of Chemistry and Physics, Louisiana State University Shreveport, LA 71115, USA \\ Correspondence should be addressed to Manhong Liu; liumanhong68@126.com and William W. Yu; wyu6000@gmail.com
}

Received 5 June 2019; Revised 19 August 2019; Accepted 29 August 2019; Published 2 December 2019

Academic Editor: Nathan C. Lindquist

Copyright (C) 2019 Ning Sui et al. This is an open access article distributed under the Creative Commons Attribution License, which permits unrestricted use, distribution, and reproduction in any medium, provided the original work is properly cited.

\begin{abstract}
A facile method to prepare $\mathrm{Pt}-\mathrm{Cu}$ nanowires (NWs) was introduced. Structural characterization such as high-resolution transmission electron microscope (HR-TEM), selected-area electron diffraction (SAED), EDS element mapping, X-ray photoelectron spectroscopy (XPS), X-ray diffraction (XRD), and inductively coupled plasma mass spectrometry (ICP-MS) showed the formation of Pt-Cu alloy, with a width of $4.5 \mathrm{~nm}$ on average. The formation process of Pt-Cu NWs was studied; it was found that bromine ion, who has preferential adsorption on Pt (100) face, served as a growth-directing agent; Brij58 not only served as a protector but also played an important role in forming Pt-Cu NWs; the mechanism was proposed. Their electrocatalytic activity towards methanol oxidation was investigated; we found that the current density of Pt-Cu NWs was $295 \mathrm{~mA} \cdot \mathrm{mg}^{-1}$ when the ratio of $\mathrm{Pt} / \mathrm{Cu}$ is $1: 1$, which is 11.5 and 2.35 times higher than that of pure $\mathrm{Pt}\left(26 \mathrm{~mA} \cdot \mathrm{mg}^{-1}\right)$ and commercial Pt/C $\left(126 \mathrm{~mA} \cdot \mathrm{mg}^{-1}\right)$. The high electrocatalytic activity is attributed to the presence of abundant structural defects and surface active sites on the synthesized Pt-Cu NWs.
\end{abstract}

\section{Introduction}

Direct methanol fuel cell (DMFC) technology is promising in energy conversion because of the rich resources, high energy density, and low pollution $[1,2]$. Platinum nanomaterials are commonly used as the anode catalysts in the methanol oxidation reaction (MOR) on account of their high electrocatalytic activity $[3,4]$. Nevertheless, the commercial application of DMFC is still limited by the high cost and low antipoisoning ability of Pt. To address these issues, substantial efforts have been devoted by partially replacing Pt with transition element $(\mathrm{Cu}, \mathrm{Co}, \mathrm{Fe}$, etc.) $[5,6]$. Among various Pt/nonnoble metal alloys, $\mathrm{Pt}$ and $\mathrm{Cu}$ have a significantly favorable synergistic effect $[7,8]$.

As we know, the tuning of the structure and morphology of nanomaterials can further improve the catalytic activity. For instance, $\mathrm{Li}$ and coworkers synthesized $\mathrm{Pt}-\mathrm{Cu}$ nanotubes, which showed attractive catalytic activity of $6.09 \mathrm{~mA} \mathrm{~cm}^{-2}$ and high stability towards MOR compared with commercial
Pt black [9]. Zhang et al. prepared diverse Pt-Cu alloy nanocrystals (dendrite, yolk-cage, and box) by changing the concentration of glycine; these catalysts with different morphology exhibited enhanced electrocatalytic performance for MOR (the highest specific activity from yolk-cage dendrite reached $2.8 \mathrm{~mA} \mathrm{~cm}^{-2}$ ) in comparison with commercial $\mathrm{Pt}$ black [10]. Lin's group reported an efficient synthetic method of porous $\mathrm{Pt}_{78} \mathrm{Cu}_{22}$ nanodendrites with superior electrocatalytic activity towards the oxygen reduction reaction [11]. Up to now, various $\mathrm{Pt}-\mathrm{Cu}$ alloyed nanostructures have been reported, including nanocage, nanoconcave, nanocube, nanosheet, hollow nanosphere, and nanowire (NW); they all showed super catalytic properties [12-14].

Ultrathin Pt-based alloy NWs with uncommon onedimensional (1D) morphology and high surface area usually exhibit excellent performance, due to their inherent anisotropy characteristic, higher structural stability, reactive surface, and easy electron transport. Accordingly, the Pt-based nanomaterials with such attractive structure have been 


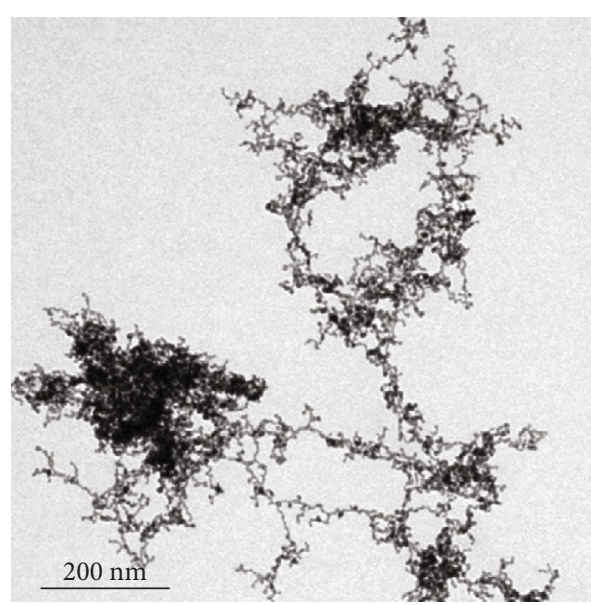

(a)

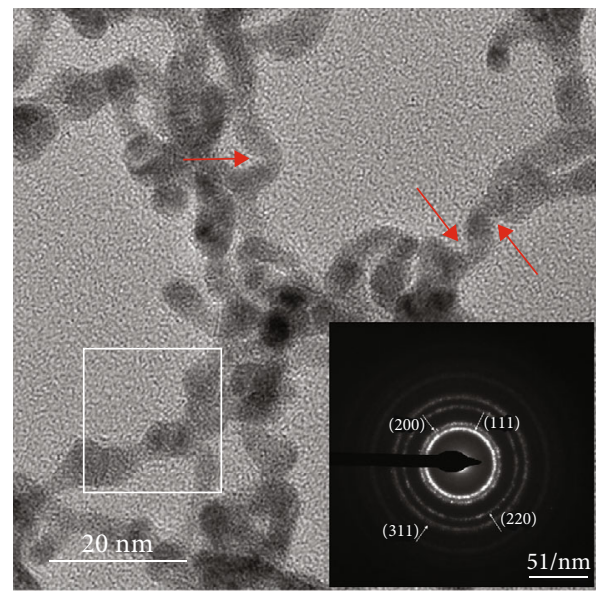

(c)

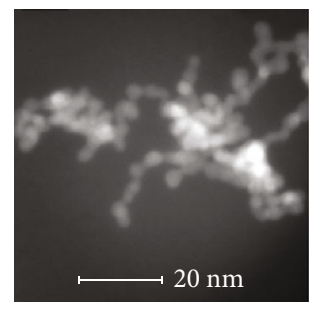

(e)

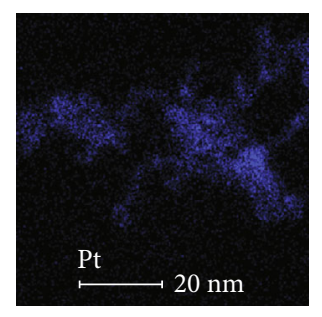

(f)

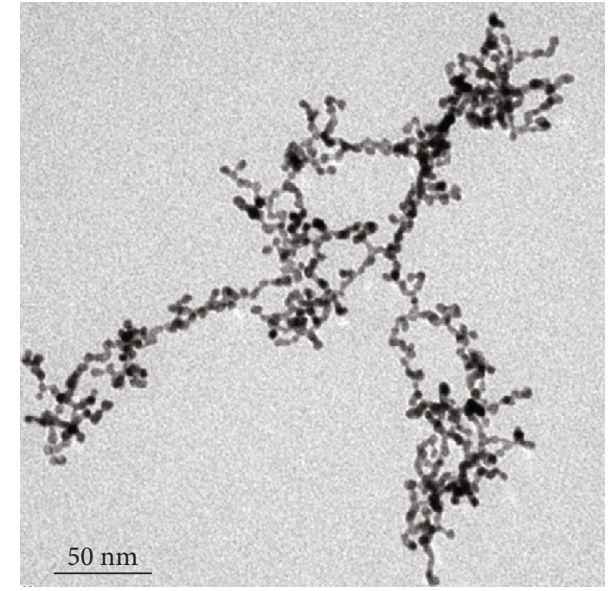

(b)

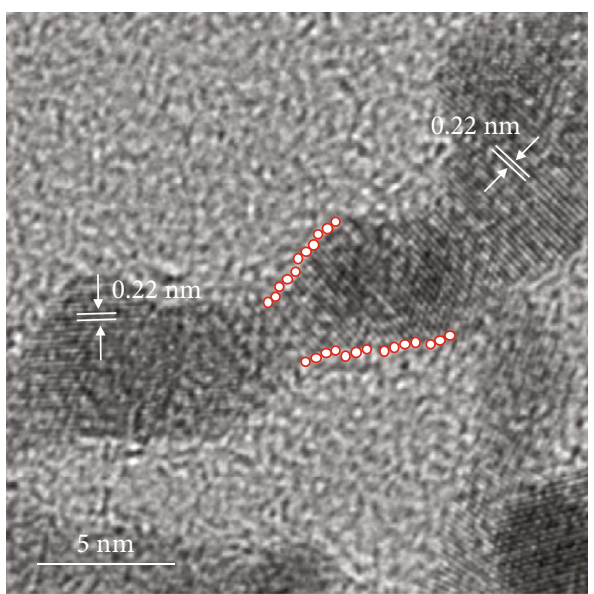

(d)

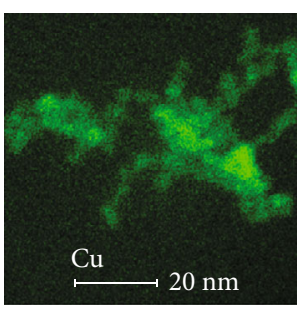

(g)

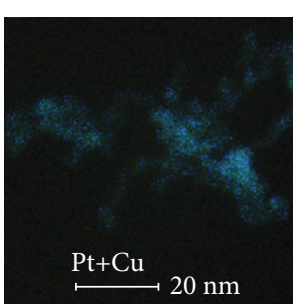

(h)

Figure 1: (a-c) Representative TEM images of ultrathin Pt-Cu NWs (the inset in Figure 1(c) shows a corresponding SAED pattern). (d) High-magnification TEM image of the square in (c). (e-h) HAADF-STEM image and the corresponding elemental maps of $\mathrm{Pt}_{1} \mathrm{Cu}_{1} \mathrm{NWs}$.

generally regarded as excellent electrocatalysts. Recently, Dai et al. reported the preparation of ultrathin Pt NWs by a solvothermal method, and these Pt NWs showed remarkable electrocatalytic activity and excellent stability in comparison to $\mathrm{Pt} / \mathrm{C}$ catalyst towards the oxidation of methanol and formic acid as well as the oxygen reduction reaction [15]. Xia's group successfully synthesized Pt-Sn NWs by a general wetchemical method, which indicated $\mathrm{Pt}_{6} \mathrm{Sn}_{3}$ with big surface area and countless defects exhibited superior activity towards MOR and ethanol oxidation reaction (EOR) [16]. Song's group reported that ultrathin $\mathrm{PtCu}_{3}$ alloy NWs were successfully obtained by introducing $\mathrm{CO}$ in the reaction. Compared with the Pt-Ru and Pt black catalysts, the electrocatalytic activity of $\mathrm{PtCu}_{3}$ for MOR increased by 1.4 -fold and 2.0 -fold, respectively [17]. Hong and coworkers reported a novel synthesis of ultrafine Pt-Cu NWs by a template-assistant method. The Pt-Ru NWs were applied in MOR and EOR, which showed excellent activity in alkaline conditions [18].

In general, Pt-based NWs were synthesized by the reduction of metal precursors in the organic compounds. Some of these solvents are not environment friendly, and some of them are difficult to remove when they attach to the surface of NWs, which could have negative influences on their catalytic activity. Consequently, it is essential to explore a simple and environment-friendly approach to synthetize Pt-based NWs in an aqueous system. Meanwhile, gram-scale synthesis 


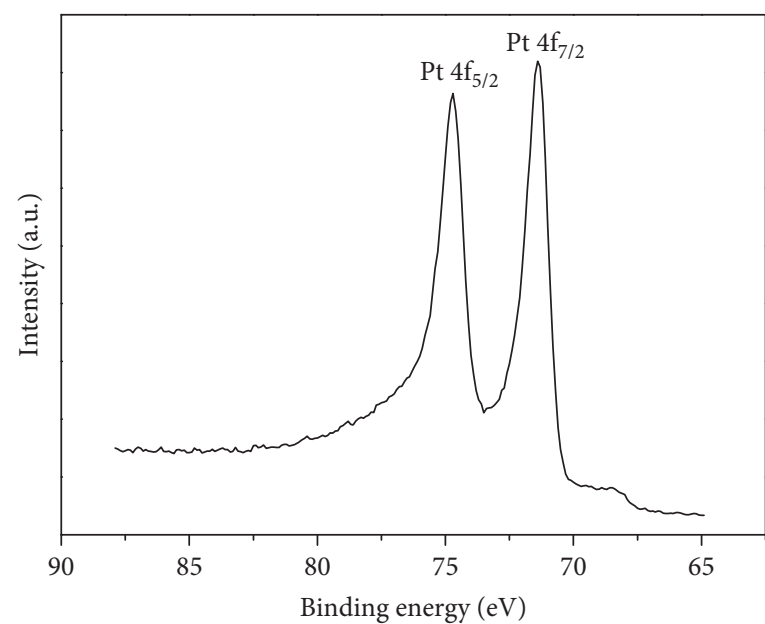

(a)

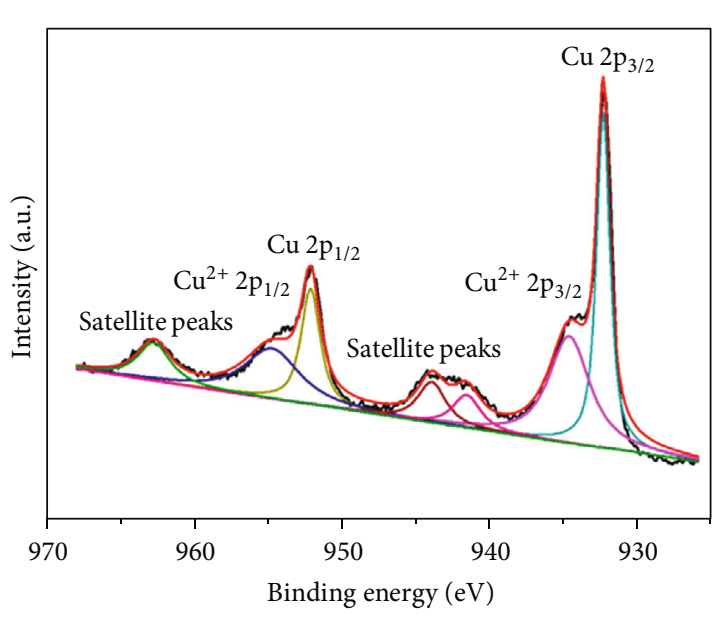

(b)

Figure 2: XPS spectra of $\mathrm{Pt}_{1} \mathrm{Cu}_{1}$ NWs: (a) Pt $4 \mathrm{f}$ and (b) Cu 2p.

with high yield is necessary to promote the commercial application of these catalysts.

To address the above issues, we developed a facile scalable synthetic strategy for the preparation of Pt-Cu NWs in an aqueous solution without any organic solvent involved. $\mathrm{Br}^{-}$and Brij58 were added to form NWs; the formation mechanism was investigated. Moreover, owing to the abundant surface atoms and substantial numbers of surface active sites, the as-prepared $\mathrm{Pt}-\mathrm{Cu}$ NWs displayed enhanced electrocatalytic activity as well as stability for MOR.

\section{Experimental}

2.1. Reagents and Chemicals. Brij58 and poly(vinyl pyrrolidone) (PVP) were supplied from Aladdin Chemical Reagent Company. Formaldehyde (HCHO) solution (40\%), methanol $\left(\mathrm{CH}_{3} \mathrm{OH}\right)$, cupric chloride dihydrate $\left(\mathrm{CuCl}_{2} \cdot 2 \mathrm{H}_{2} \mathrm{O}\right)$, chloroplatinic acid hexahydrate $\left(\mathrm{H}_{2} \mathrm{PtCl}_{6} \cdot 6 \mathrm{H}_{2} \mathrm{O}\right)$, sodium fluoride $(\mathrm{NaF})$, sodium chloride $(\mathrm{NaCl})$, sodium bromide $(\mathrm{NaBr})$, and sodium iodide $(\mathrm{NaI})$ were purchased from Sinopharm Chemical Reagent Co., Ltd. All the materials were used directly upon receiving, and lab-prepared deionized water was used.

2.2. Synthesis of Ultrafine Pt-Cu NWs. In a typical synthesis, $160 \mathrm{mg}$ of Brij58 and $154.8 \mathrm{mg}$ of $\mathrm{NaBr}$ were added into $10 \mathrm{~mL}$ of deionized water with stirring at room temperature until Brij58 was completely dissolved. After that, $2.5 \mathrm{~mL}$ of $\mathrm{H}_{2} \mathrm{PtCl}_{6} \cdot 6 \mathrm{H}_{2} \mathrm{O}(20 \mathrm{mM})$ and $2.5 \mathrm{~mL}$ of $\mathrm{CuCl}_{2} \cdot 2 \mathrm{H}_{2} \mathrm{O}$ $(20 \mathrm{mM})$ solutions were introduced. The mixed solution was continuously stirred for 15 minutes, then $680 \mu \mathrm{L}$ of formaldehyde (40\%) was added. The reaction mixture was transferred into a $20 \mathrm{~mL}$ Teflon-lined stainless-steel autoclave. The sealed vessel was heated under $200^{\circ} \mathrm{C}$ for $6 \mathrm{~h}$, and it was cooled naturally to room temperature. Finally, the resulting products were collected by centrifugation $(8000 \mathrm{rpm}$ for $10 \mathrm{~min}$ ) and washed with water/ethanol for several times. The obtained samples were dispersed in ethanol for further use.

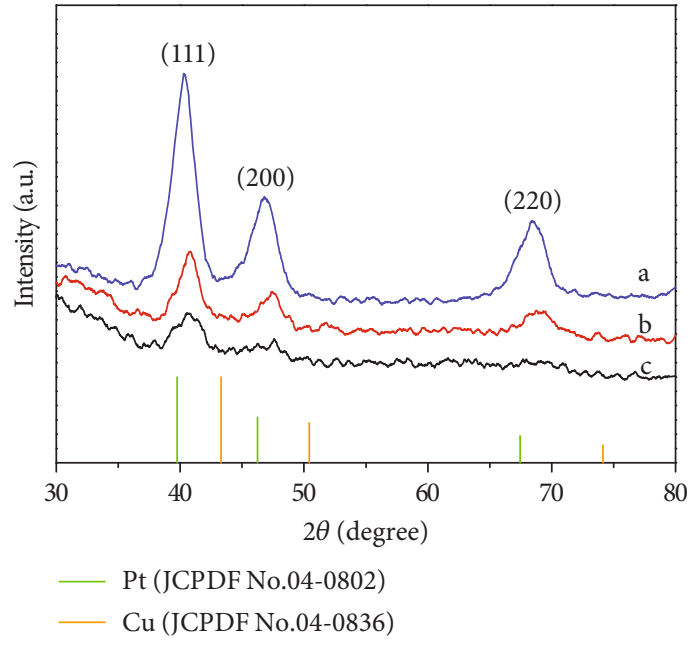

Figure 3: XRD patterns of Pt-Cu NWs: (a) $\mathrm{Pt}_{4} \mathrm{Cu}_{1}$, (b) $\mathrm{Pt}_{1} \mathrm{Cu}_{1}$, and (c) $\mathrm{Pt}_{1} \mathrm{Cu}_{4}$.

2.3. Material Characterization. Transmission electron microscopy (TEM) images were determined with an H-7650 microscope operating at $200 \mathrm{kV}$. HRTEM images and EDS elemental mappings were recorded using an FEI Tecnai F20 FEG-TEM microscope operated at $200 \mathrm{kV}$. X-ray diffraction (XRD) data were determined by a D-MAX 2500/PC diffractometer. X-ray photoelectron spectroscopy (XPS) analysis was acquired on an ESCALAB 250 spectrometer with $\mathrm{Al} \mathrm{K} \alpha \mathrm{X}$-ray excitation. ICP-MS analysis of samples was performed on NexION 350D. The electrochemical tests were carried out by using a CHI $660 \mathrm{E}$ electrochemical analyzer at room temperature.

\section{Results and Discussion}

Figures 1(a)-1(c) displayed the typical TEM images of the as-prepared $\mathrm{Pt}-\mathrm{Cu}$ NWs under different magnifications. These NWs were curved and interlace with each other for 


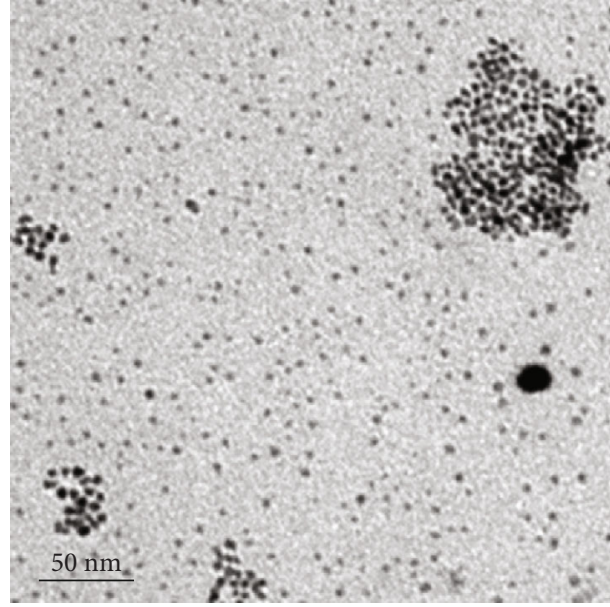

(a)

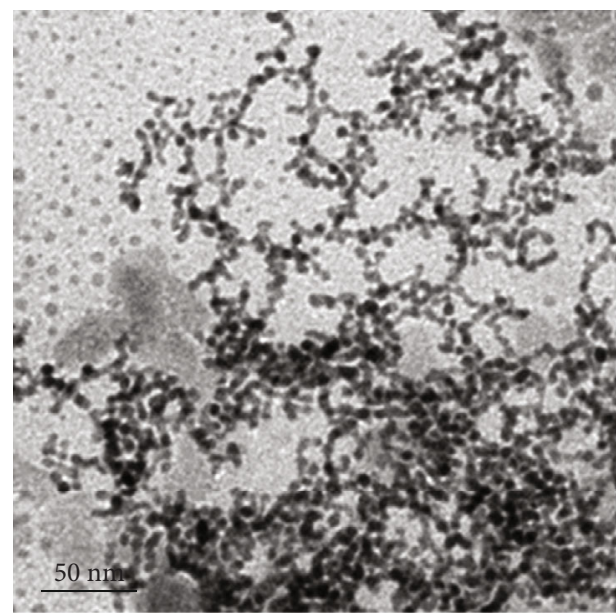

(c)

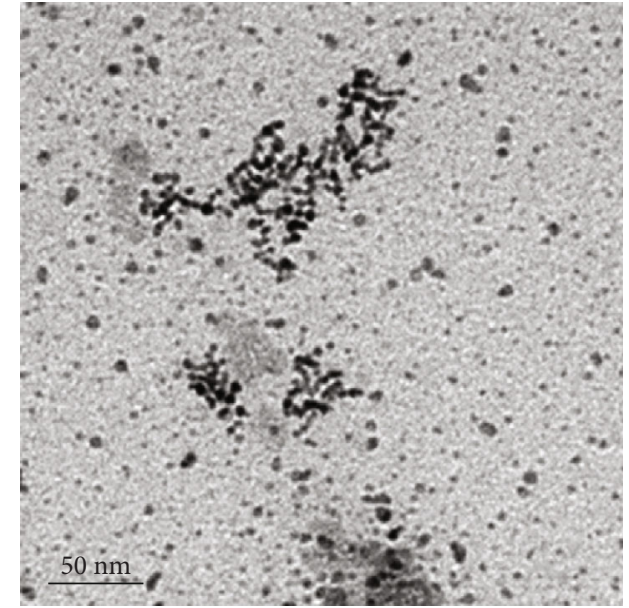

(b)

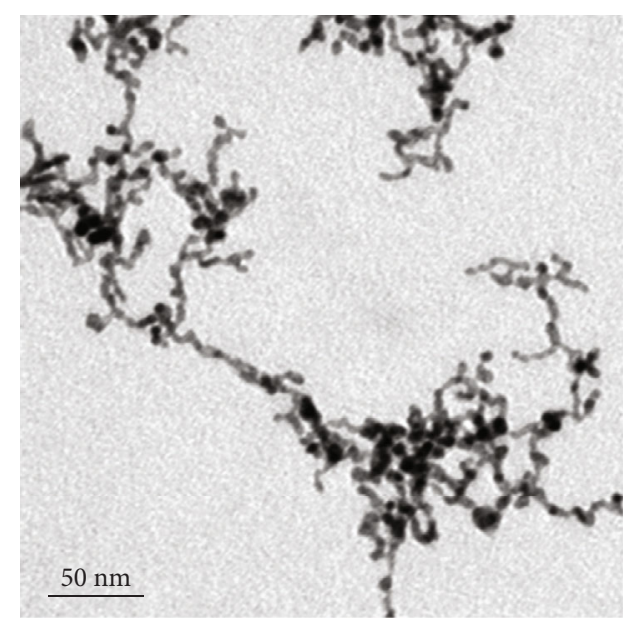

(d)

Figure 4: TEM images of intermediates acquired with $\mathrm{Pt}_{1} \mathrm{Cu}_{1}$ alloy NWs at different stages: (a) $30 \mathrm{~min}$, (b) $60 \mathrm{~min}$, (c) $120 \mathrm{~min}$, and (d) $8 \mathrm{~h}$, respectively.

the formation of NWs networks, indicating their highly flexible feature. The average diameter of the NWs was around $4.5 \mathrm{~nm}$. The $\mathrm{Pt} / \mathrm{Cu}$ atomic ratio determined by the ICP-MS measurement was 45:55 (Table S1), which was in accord with the $\mathrm{Pt} / \mathrm{Cu}$ atomic ratio in the initial reaction system.

The selected-area electron diffraction (SAED) image of an individual Pt-Cu NWs (Figure 1(c), inset) displayed that the NWs were polycrystals and the diffraction rings were in good agreement with (111), (200), (220), and (311) planes of the Pt-Cu alloy phase. The high-resolution TEM (HRTEM) image of crystal grains with their lattice orientation (selected from the square area in Figure 1(c)) is shown in Figure 1(d). The interplanar spacing of $0.22 \mathrm{~nm}$ was clearly observed, which was smaller than the (111) lattice spacing $(0.226 \mathrm{~nm})$ of Pt crystal but larger than the (111) lattice spacing (0.208) of $\mathrm{Cu}$. This confirmed the production of a $\mathrm{Pt}-\mathrm{Cu}$ alloy. In addition, there were abundant grain boundaries along with the Pt-Cu NWs networks, as indicated by red arrows in Figure 1(c). Moreover, many structural defects (such as atomic steps, corner and edge atoms) were observed on the surface (marked with red circles in Figure $1(\mathrm{~d})$ ). It has been researched that these structural defects could serve as active catalytic sites to enhance the catalytic activity effectively $[19,20]$. The elemental mapping showed these NWs were composed of $\mathrm{Pt}$ and $\mathrm{Cu}$, and the two elements were homogeneously distributed over the whole NWs (Figures 1(e)-1(h)).

In order to investigate the surface composition and valence state of Pt-Cu NWs, XPS measurements were used for further characterization (Figure 2). The Pt $4 \mathrm{f}$ spectrum exhibits peaks at $74.7 \mathrm{eV}$ and $71.4 \mathrm{eV}$, which could be originated in $\mathrm{Pt} 4 \mathrm{f}_{7 / 2}$ and $\mathrm{Pt} 4 \mathrm{f}_{5 / 2}$ of metallic $\mathrm{Pt}^{0}$ [21], respectively. The $\mathrm{Cu} 2 \mathrm{p}$ spectrum exhibited that metallic $\mathrm{Cu}^{0}(932.2 \mathrm{eV})$ was predominant while a weak signal of $\mathrm{Cu}^{2+}(\sim 934.5)$ also exists. The existence of tiny amount of $\mathrm{Cu}^{2+}$ could be further confirmed by a satellite peak at $\sim 943 \mathrm{eV}$, which was attributed to the oxidation of surface $\mathrm{Cu}$ atoms in air. An obvious positive shifted $(+0.2 \mathrm{eV})$ in the binding energy of $\mathrm{Pt} 4 \mathrm{f}_{7 / 2}$ was presented compared to pure $\mathrm{Pt}(71.2 \mathrm{eV})$. The shift was probably originated in the changes of the electronic structure by the charge transfer between $\mathrm{Pt}$ and $\mathrm{Cu}$.

In addition, different Pt-Cu alloy $\mathrm{NWs}$ were obtained by adjusting the ratio of $\mathrm{Pt}^{4+}$ to $\mathrm{Cu}^{2+}$ (Fig. S1). The width of well-dispersed wavy NWs slightly increased from 4.0 to 


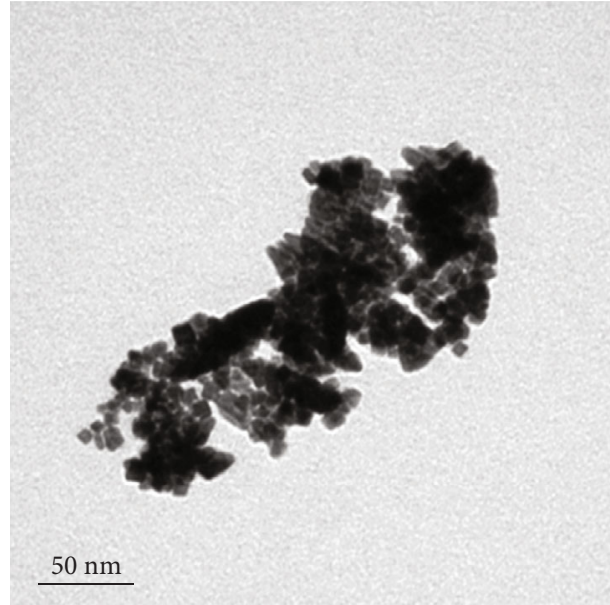

(a)

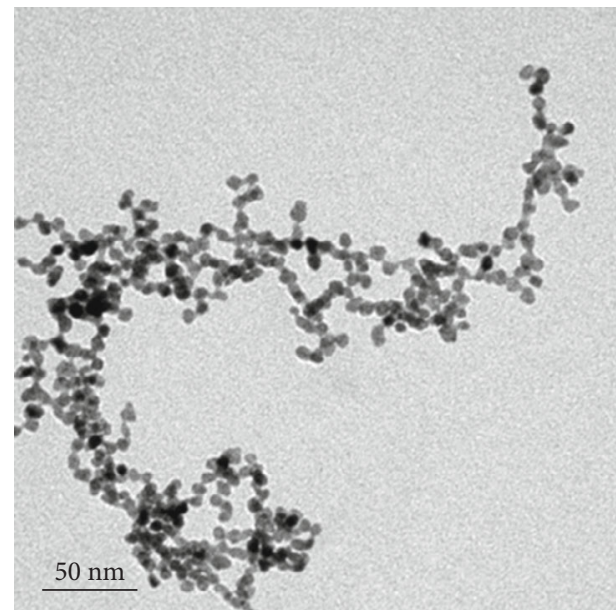

(c)

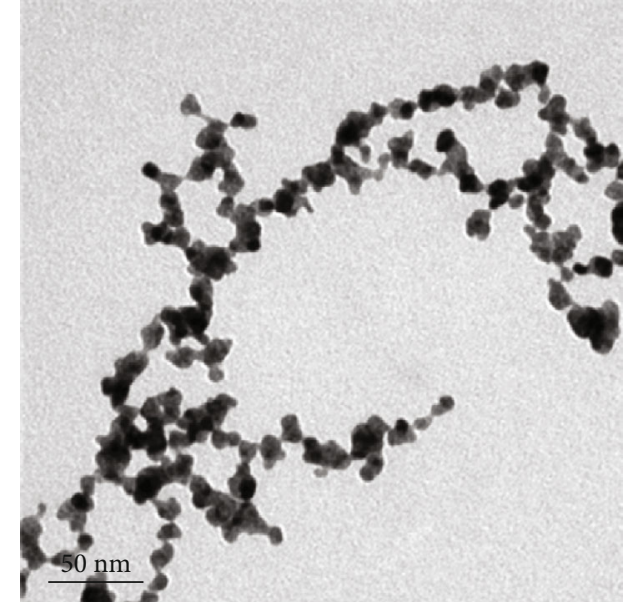

(b)

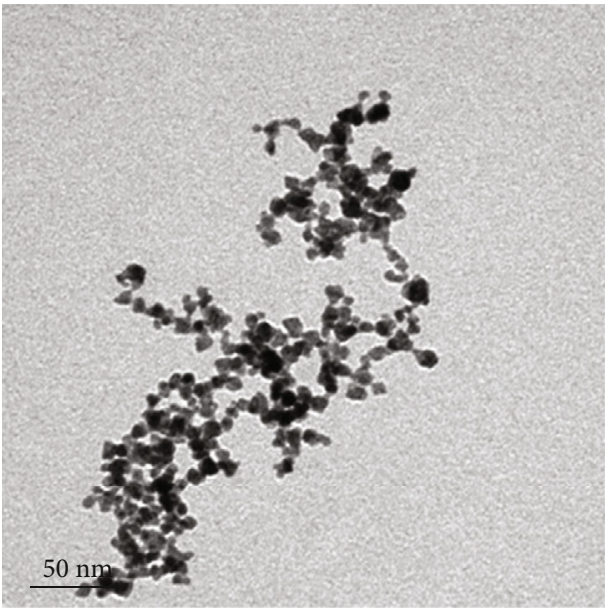

(d)

FIgURE 5: (a) TEM images of $\mathrm{Pt}_{1} \mathrm{Cu}_{1}$ nanoparticles synthesized without any halogen ions and in the presence of (b) $\mathrm{F}^{-}$, (c) $\mathrm{Cl}^{-}$, and (d) $\mathrm{I}^{-}$.

$4.9 \mathrm{~nm}$ with the increase of the $\mathrm{Pt} / \mathrm{Cu}$ ratio. Shevchenko's group has demonstrated the size of $\mathrm{CoPt}_{3}$ nanocrystals shrank with the decrease of the Pt/Co ratio, which could be attributed to an increase of the nucleation rate [22]. Similarly, under the current system, the decrease of the $\mathrm{Pt} / \mathrm{Cu}$ ratio led to the reduction of the width of Pt-Cu NWs, which might be explained by the decrease of nucleation rate. With the low content of $\mathrm{Cu}$ precursor, fewer nucleation seeds were generated and therefore the wider Pt-Cu NWs were formed. Additionally, the uniform nanowire network could not be formed in the absence of $\mathrm{Cu}^{2+}$ and nanoparticles with obvious aggregation were obtained, as shown in Fig. S2. The results demonstrated a key role of $\mathrm{Cu}$ precursor in facilitating the formation of Pt-Cu NWs.

The crystalline structures of these Pt-Cu NWs were detected by X-ray diffraction (XRD). As shown in Figure 3, the typical diffraction peaks of a face-centered-cubic structure could be observed. Three diffraction peaks were originated from the (111), (200), and (220) planes of Pt-Cu NWs; all peaks were situated between the standard peaks of Pt (JCPDS no. 04-0802) and $\mathrm{Cu}$ (JCPDS no. 04-0836), which was indicative of the formation of an alloyed structure $[23,24]$. The diffraction peaks of all samples were shifted to smaller angle with the increase of the ratio of $\mathrm{Pt} / \mathrm{Cu}$, further confirming the formation of alloyed Pt-Cu nanocrystals [25]. Moreover, all diffraction peaks were widened due to the ultrathin feature of NWs.

In order to demonstrate the influence of bromine ions, a series of control experimentation was performed. When $\mathrm{NaBr}$ was absent while the other experimental conditions were maintained, as exhibited in Figure 4(a), irregular particles with severe aggregation were obtained. When $\mathrm{Br}^{-}$was replaced by $\mathrm{F}^{-}, \mathrm{Cl}^{-}$, and $\mathrm{I}^{-}$, discontinuous and inhomogeneous nanochains were formed with an average size of $14 \mathrm{~nm}, 7 \mathrm{~nm}$, and $10 \mathrm{~nm}$, respectively, as shown in Figures 4(b)-4(d). The result further confirmed the essentiality of $\mathrm{Br}^{-}$in this experiment system. The different morphologies could be ascribed to the following reasons. Firstly, when $\mathrm{H}_{2} \mathrm{PtCl}_{6} \cdot 6 \mathrm{H}_{2} \mathrm{O}$ precursor was introduced as a solution containing bromine ions, $\left[\mathrm{PtBr}_{6}\right]^{2-}$ would become foremost ingredients due to the stronger coordination between $\mathrm{Br}^{-}$and $\mathrm{Pt}^{4+}$ [26-28], thus improving the stability and reducing the reaction rate. Secondly, the preferential adsorption of bromine ion on Pt (100) face would restrict 


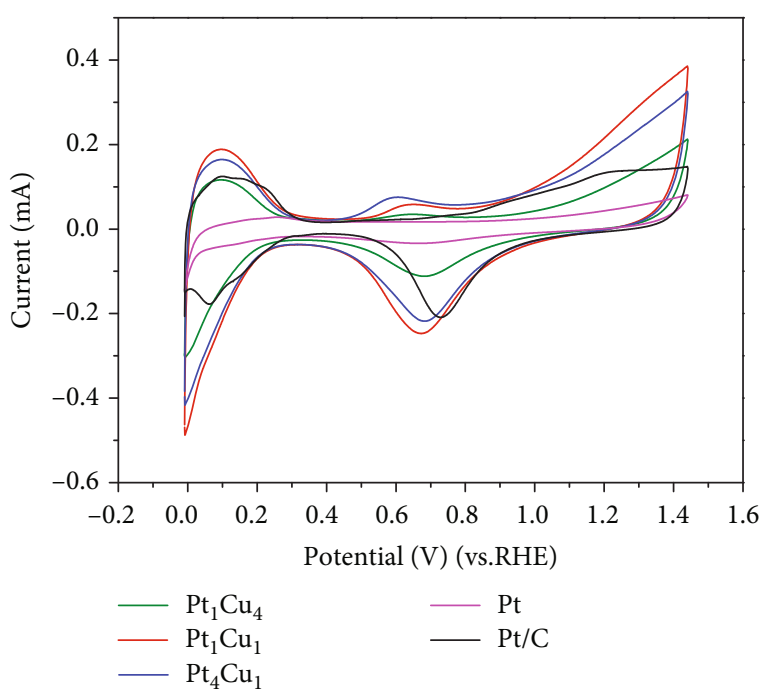

(a)

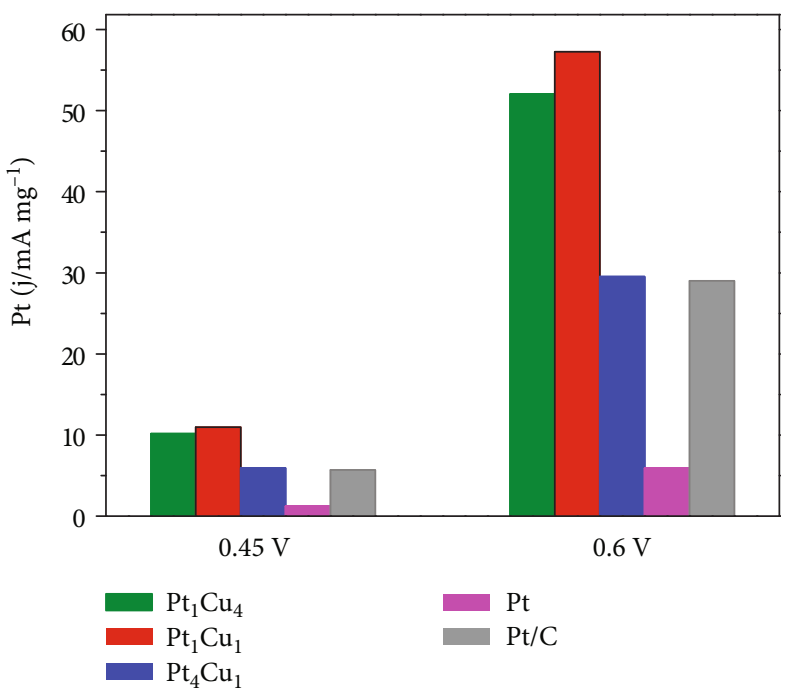

(c)

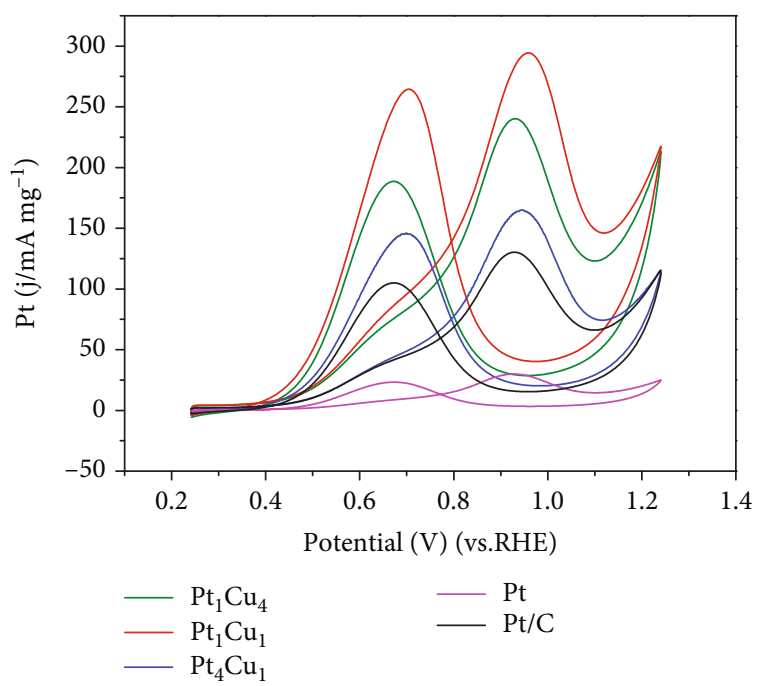

(b)

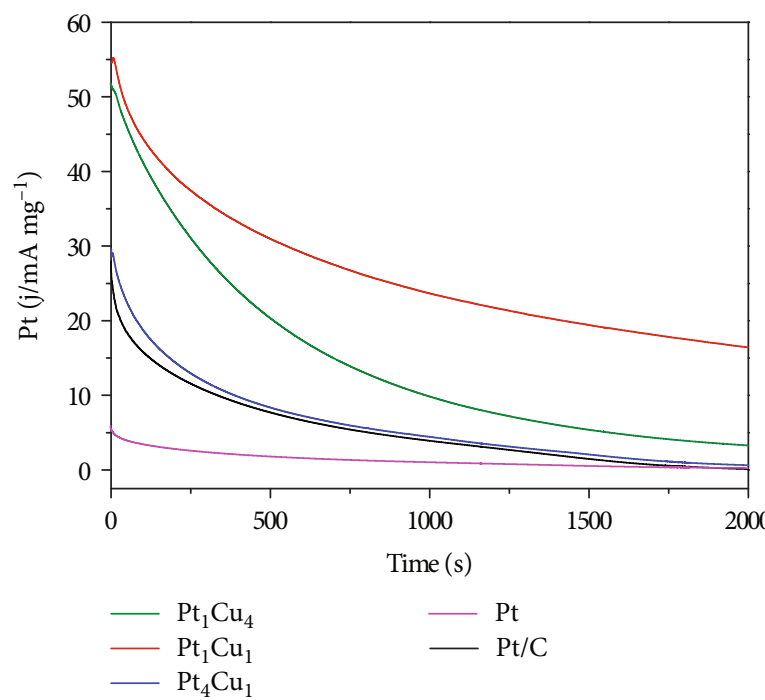

(d)

Figure 6: Cyclic voltammetry curve of five different catalysts in (a) $0.5 \mathrm{M} \mathrm{H}_{2} \mathrm{SO}_{4}$ and (b) $0.5 \mathrm{M} \mathrm{H}_{2} \mathrm{SO}_{4}+1 \mathrm{M} \mathrm{CH}_{3} \mathrm{OH}$ for $\mathrm{MOR}$ at a sweep rate of $50 \mathrm{mV} \mathrm{s}^{-1}$. (c) The histogram of catalytic activities of these five catalysts at 0.45 and $0.6 \mathrm{~V}$. (d) CA curves recorded at $0.6 \mathrm{~V}$ for $\mathrm{MOR}$ in $0.5 \mathrm{M}$ $\mathrm{H}_{2} \mathrm{SO}_{4}$ containing $1 \mathrm{M} \mathrm{CH}_{3} \mathrm{OH}$.

the growth of this face $[29,30]$, and this bonding between platinum and bromine ions benefited the anisotropic growth of the Pt-Cu NWs.

The surfactant of Brij58 micelles also played an important role in the formation of NWs. In our system, metalaqua complexes could be formed when metallic precursors were coordinated by water molecules. These complexes were adsorbed on the external hydrophilic $\mathrm{CH}_{2} \mathrm{CH}_{2} \mathrm{O}$ region of the Brij58 micelles owing to the interaction between the $\mathrm{CH}_{2} \mathrm{CH}_{2} \mathrm{O}$ group and the coordinated water molecules [31]. While there was no surfactant involved in the reaction system, the nanoparticles obtained were heavily agglomerated with indefinite shapes, as shown in Fig. S3a. These results demonstrated that a role of Brij58 was played to prevent the crystal from agglomeration. PVP is a very common surface protector in order to well disperse noble metal nano- structures [32-34]. When PVP was used, nanoparticles with a diameter of about $3 \mathrm{~nm}$ were obtained without NWs (Fig. S3b). It confirmed that Brij58 not only served as a stabilizing agent but also had a crucial effect on the formation of the Pt-Cu NWs.

The intermediates at different growth stages were characterized by TEM in order to investigate the growth mechanism of $\mathrm{Pt}-\mathrm{Cu} \mathrm{NWs}$ (Figure 5). At the early stage of the reaction $(30 \mathrm{~min}),\left[\mathrm{PtBr}_{6}\right]^{2-}$ and $\mathrm{Cu}^{2+}$ were reduced quickly by formaldehyde, and substantial sub- $4 \mathrm{~nm}$ nanoparticles were observed. After $1 \mathrm{~h}$, some nanoparticles began to coalesce with each other and some short NWs formed. After $2 \mathrm{~h}$, a large number of $\mathrm{Pt}-\mathrm{Cu}$ alloy NWs were obtained in virtue of continuous attachment. The pleasant surprise was the width of NWs remained roughly constant in the process of reaction. Furthermore, the obvious nodes were present in 


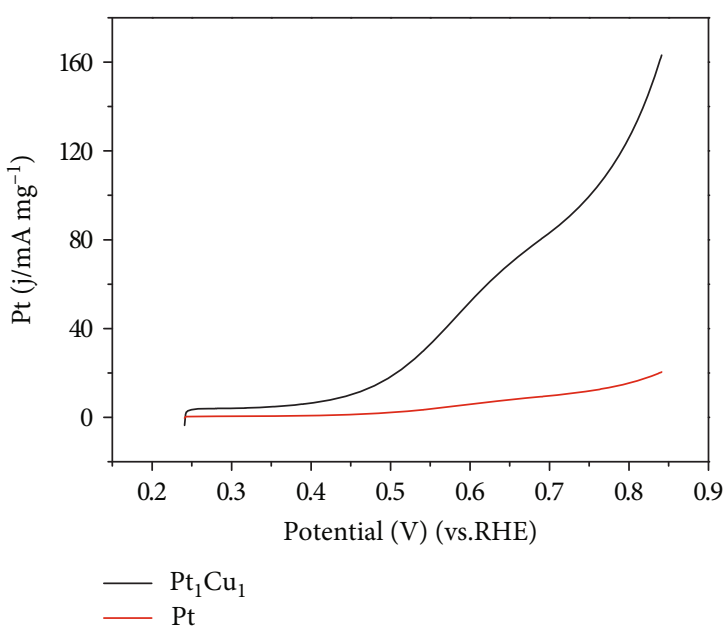

(a)

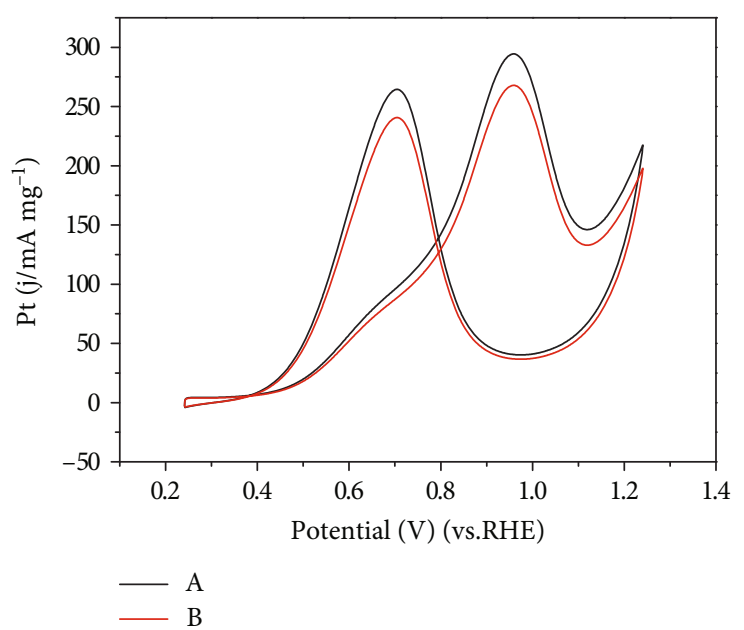

(b)

FIguRe 7: (a) LSV curves for MOR over $\mathrm{Pt}_{1} \mathrm{Cu}_{1} \mathrm{NWs}$ and pure Pt in $0.5 \mathrm{M} \mathrm{H}_{2} \mathrm{SO}_{4}$ and $1 \mathrm{M} \mathrm{CH}_{3} \mathrm{OH}$. (b) CV curves for $\mathrm{MOR}_{\text {over }} \mathrm{Pt}_{1} \mathrm{Cu} \mathrm{NW}_{1} \mathrm{NW}$ (A) fresh electrode, (B) the same electrode saved for a week.

the Pt-Cu NWs (Figure 1(c)) which further indicated that the lattice-matched oriented attachment growth mechanism is appropriate in the reaction system [35]. Figure 5(d) showed the nanocrystals after $8 \mathrm{~h}$. Compared with the production of $6 \mathrm{~h}$ (Figure 1(b)), there was no obvious variations in morphology and size, demonstrating that the reaction finished when the reaction time was extended to $6 \mathrm{~h}$. The synthesis strategy was general and scalable. We tried to scale it up by 10 times, and the Pt-Cu NWs were well-dispersed with the same morphology (Fig. S4).

The as-prepared NWs with different compositions of $\mathrm{Pt}_{1} \mathrm{Cu}_{4}, \mathrm{Pt}_{1} \mathrm{Cu}_{1}$, and $\mathrm{Pt}_{4} \mathrm{Cu}_{1}$ were conducted to evaluate their catalytic performances of MOR. For comparison, pure $\mathrm{Pt}$ nanoparticles and commercial $\mathrm{Pt} / \mathrm{C}$ were also tested as the reference catalysts. The cyclic voltammogram (CV) was firstly carried out in $0.5 \mathrm{M} \mathrm{H}_{2} \mathrm{SO}_{4}$ from $-0.25 \mathrm{~V}$ to $0.2 \mathrm{~V}$ (Figure 6(a)). The electrochemically surface active area (ECSA) of $\mathrm{Pt}_{1} \mathrm{Cu}_{4}, \mathrm{Pt}_{1} \mathrm{Cu}_{1}, \mathrm{Pt}_{4} \mathrm{Cu}_{1}, \mathrm{Pt}$, and commercial $\mathrm{Pt} / \mathrm{C}$ after calculation were $86.8,93.7,66.3,11.2$, and $52.5 \mathrm{~m}^{2} \mathrm{~g}^{-1}$, respectively. Figure 6(b) presented the $\mathrm{CV}$ curves of five catalysts in a mixture of $0.5 \mathrm{M} \mathrm{H}_{2} \mathrm{SO}_{4}$ and $1 \mathrm{M} \mathrm{CH}_{3} \mathrm{OH}$. Apparently, the CV curves displayed two anodic peaks which were the methanol oxidation peak (at the higher potential) and the intermediate carbonaceous species oxidation peak (at the lower potential). The mass activity of electrocatalysts was usually taken as an index to evaluate their activity. The peak current density of $\mathrm{Pt}_{1} \mathrm{Cu}_{4}, \mathrm{Pt}_{1} \mathrm{Cu}_{1}, \mathrm{Pt}_{4} \mathrm{Cu}_{1}, \mathrm{Pt}$, and commercial $\mathrm{Pt} / \mathrm{C}$ were $235,295,164,127$, and $29 \mathrm{~mA} \cdot \mathrm{mg}^{-1}$, respectively. As displayed in Figure 6(c), the current density at lower potential 0.45 and $0.6 \mathrm{~V}$ (vs. RHE) of $\mathrm{Pt}_{1} \mathrm{Cu}_{1}$ NWs was the highest (11 and $57 \mathrm{~mA} \cdot \mathrm{mg}^{-1}$, respectively), which was higher than that of $\mathrm{Pt}_{1} \mathrm{Cu}_{4}\left(10.16\right.$ and $\left.52.02 \mathrm{~mA} \cdot \mathrm{mg}^{-1}\right), \mathrm{Pt}_{4} \mathrm{Cu}_{1}(5.92$ and $\left.29.54 \mathrm{~mA} \cdot \mathrm{mg}^{-1}\right)$, Pt $\left(1.23\right.$ and $\left.5.89 \mathrm{~mA} \cdot \mathrm{mg}^{-1}\right)$, and $\mathrm{Pt} / \mathrm{C}$ (5.69 and $\left.28.99 \mathrm{~mA} \cdot \mathrm{mg}^{-1}\right)$. Compared with pure Pt, $\mathrm{Pt}_{1} \mathrm{Cu}_{1}$ NWs had lower onset potential, which was beneficial for MOR on the surface of Pt-Cu NWs (Figure 7(a)). The stability of the catalysts was further measured by repeating the potential for $0.6 \mathrm{~V}$ (vs. $\mathrm{RHE}$ ). $\mathrm{Pt}_{1} \mathrm{Cu}_{1}$ catalyst showed the best stability among the five catalysts during a 2000 s test, as shown in Figure 6(d). Compared with Pt black and pure Pt, the Pt-Cu NWs displayed bigger oxidation current and slower current decay rate, demonstrating a good stability. Additionally, the same electrode was saved for a week for reuse, and its current density presented $91 \%$ of the initial value (Figure 7(b)), which further demonstrates their excellent reusability.

The activity of the catalyst decreased with the time in the stability test. There might be two reasons [36, 37]: (1) as this process goes on continuously, the catalyst morphology changed; (2) at the beginning of the potential scan, a rapid accumulation of strongly adsorbed species (CO, oxygenate, etc.) occurred on the catalyst surface, which caused the number of active sites to decrease rapidly. Taking $\mathrm{Pt}_{1} \mathrm{Cu}_{1} \mathrm{NWs}$ as an example, their TEM characterization after the stability test showed that there was no obvious change in the morphology of the catalyst (Fig. S5a), which proved that the decrease of catalyst activity was not caused by the change of catalyst morphology. Then, the CO stripping experiment was carried out on the electrode after the stability test (Fig. S5b). It was found that the catalyst had obvious CO stripping peak $(870 \mathrm{mV})$, indicating the accumulation of $\mathrm{CO}$ on the surface of $\mathrm{Pt}_{1} \mathrm{Cu}_{1}$ NWs during the methanol oxidation reaction, which led to the decrease of the activity of the catalyst.

The high activity and stability of Pt-Cu NWs for MOR are rationally attributed to their catalytic composition and unique structure, which could be explained as follows. (1) It is universally known that representative procedures for MOR can produce poisonous intermediates (e.g., $\mathrm{CO}$ ), which has a strong adsorption on Pt surface and reduces the catalytic performance of the catalyst. Owing to the d-band center theory [38], the $\mathrm{Cu}$ atoms in the $\mathrm{Pt}-\mathrm{Cu}$ alloy change the electronic energy level structure of Pt. Such change weakens the adsorption strength between Pt and intermediates, which is beneficial to the MOR. Meanwhile, the ability of CO adhering to $\mathrm{Cu}$ takes precedence over $\mathrm{Pt}$ which release more $\mathrm{Pt}$ atom active sites than the one without $\mathrm{Cu}$. (2) 1D NWs provide 
abundant surface atoms, structural defects (e.g., grain boundaries), and substantial numbers of surface active sites, which increase the availability of Pt atoms and enhance catalytic performance. (3) The NW's inherent anisotropic structure makes the catalysts less accessible to Ostwald ripening as well as aggregation and thus preserves their enhanced stability.

\section{Conclusions}

In conclusion, 1D Pt-Cu NWs have been acquired by an environmentally friendly and facile aqueous solution method in gram scale. The employments of $\mathrm{Br}^{-}$and Brij58 as growthdirection and stabilization reagents were considered vital for achieving Pt-Cu NWs. Compared with commercial Pt black, the as-prepared $\mathrm{Pt}_{1} \mathrm{Cu}_{1}$ NWs showed excellent activity $\left(295 \mathrm{~mA} \cdot \mathrm{mg}^{-1}\right)$ and stability towards MOR. These remarkable catalytic performances were mainly attributed to the exceptional structure with abundant structural defects and surface active sites.

\section{Data Availability}

The data used to support the findings of this study are available from the corresponding authors upon request.

\section{Conflicts of Interest}

The authors declare that they have no conflicts of interest.

\section{Acknowledgments}

This work was financially supported by the National Natural Science Foundation of China (21501106), the Scientific Research Foundation for the Returned Overseas Chinese Scholars and Qingdao Municipal Science and Technology Commission (16-5-1-86-jch), and the Chemistry Faculty Talents Foundation of Qingdao University of Science and Technology.

\section{Supplementary Materials}

Fig. S1: TEM images of Pt-Cu NWs with different $\mathrm{Pt} / \mathrm{Cu}$ ratios, (a) $1: 4$, (b) $4: 1$. Fig. S2: TEM image of Pt nanoparticles. Fig. S3: (a) TEM image of Pt-Cu alloy synthesized without addition of any surfactant, (b) TEM image of Pt-Cu nanoparticles obtained by adding PVP. Fig. S4: (a) photos of the autoclave $(200 \mathrm{~mL}$ volume) for the synthesis and the resulting product of the NWs. (b) TEM image of the gram-scale synthesis of Pt-Cu NWs. Fig. S5: (a) TEM characterization of the Pt1Cu1 catalyst after the stability test; (b) CO-stripping voltammetry of Pt1Cu1 catalyst after the stability test. Table S1: elemental analysis of the Pt-Cu NWs analyzed by the ICP-MS. Table S2: comparison of mass activity based on Pt-based catalysts in $0.5 \mathrm{M} \mathrm{H}_{2} \mathrm{SO}_{4}$ and $1 \mathrm{M} \mathrm{CH} \mathrm{CH}_{3} \mathrm{OH}$. (Supplementary Materials)

\section{References}

[1] G. Zhang, Z. Yang, C. Huang, W. Zhang, and Y. Wang, "Small-sized and highly dispersed Pt nanoparticles loading on graphite nanoplatelets as an effective catalyst for methanol oxidation," Nanoscale, vol. 7, no. 22, pp. 10170-10177, 2015.

[2] H. Lv, T. Peng, P. Wu, M. Pan, and S. Mu, "Nano-boron carbide supported platinum catalysts with much enhanced methanol oxidation activity and CO tolerance," Journal of Materials Chemistry, vol. 22, no. 18, pp. 9155-9160, 2012.

[3] V. Selvaraj, R. Thamil Magal, and D. Prasanna, "Development of platinum and platinum-tin deposited nitrogen doped novel copolymer-carbon nanotubes electrocatalyst for alcohol oxidation," Materials Research Innovations, vol. 21, no. 4, pp. 222-231, 2016.

[4] J. Wu and H. Yang, "Platinum-based oxygen reduction electrocatalysts," Accounts of Chemical Research, vol. 46, no. 8, pp. 1848-1857, 2013.

[5] X. Zhao, J. Zhang, L. Wang, H. X. Li, Z. Liu, and W. Chen, "Ultrathin PtPdCu nanowires fused porous architecture with 3D molecular accessibility: an active and durable platform for methanol oxidation," ACS Applied Materials \& Interfaces, vol. 7, no. 47, pp. 26333-26339, 2015.

[6] Y. Kuang, Z. Cai, Y. Zhang et al., "Ultrathin dendritic Pt3Cu triangular pyramid caps with enhanced electrocatalytic activity," ACS Applied Materials \& Interfaces, vol. 6, no. 20, pp. 17748-17752, 2014.

[7] Y. Qi, T. Bian, S. I. Choi et al., "Kinetically controlled synthesis of $\mathrm{Pt}-\mathrm{Cu}$ alloy concave nanocubes with high-index facets for methanol electro-oxidation," Chemical Communications, vol. 50, no. 5, pp. 560-562, 2014.

[8] M. Yang, Q. Cai, C. Liu et al., "Monodispersed hollow platinum nanospheres: facile synthesis and their enhanced electrocatalysis for methanol oxidation," Journal of Materials Chemistry A, vol. 2, no. 33, pp. 13738-13743, 2014.

[9] H. H. Li, Q. Q. Fu, L. Xu et al., "Highly crystalline PtCu nanotubes with three dimensional molecular accessible and restructured surface for efficient catalysis," Energy \& Environmental Science, vol. 10, no. 8, pp. 1751-1756, 2017.

[10] Z. Zhang, Y. Yang, F. Nosheen et al., "Fine tuning of the structure of $\mathrm{Pt}-\mathrm{Cu}$ alloy nanocrystals by glycine-mediated sequential reduction kinetics," Small, vol. 9, no. 18, pp. 3063-3069, 2013.

[11] S. Fu, C. Zhu, Q. Shi, H. Xia, D. du, and Y. Lin, "Highly branched $\mathrm{PtCu}$ bimetallic alloy nanodendrites with superior electrocatalytic activities for oxygen reduction reactions," Nanoscale, vol. 8, no. 9, pp. 5076-5081, 2016.

[12] B. Y. Xia, H. B. Wu, X. Wang, and X. W. (. D.). Lou, "One-pot synthesis of cubic PtCu3 nanocages with enhanced electrocatalytic activity for the methanol oxidation reaction," Journal of the American Chemical Society, vol. 134, no. 34, pp. 1393413937, 2012.

[13] Y. Jia, Y. Jiang, J. Zhang et al., "Unique excavated rhombic dodecahedral PtCu3 alloy nanocrystals constructed with ultrathin nanosheets of high-energy $\{110\}$ facets," Journal of the American Chemical Society, vol. 136, no. 10, pp. 3748-3751, 2014.

[14] X. Yu, D. Wang, Q. Peng, and Y. Li, "High performance electrocatalyst: Pt-Cu hollow nanocrystals," Chemical Communications, vol. 47, no. 28, pp. 8094-8096, 2011.

[15] L. Dai, S. Mo, Q. Qin, X. Zhao, and N. Zheng, "Carbon Monoxide-Assisted Synthesis of Ultrathin PtCu3Alloy Wavy Nanowires and Their Enhanced Electrocatalysis," Small, vol. 12, no. 12, pp. 1572-1577, 2016. 
[16] B. Y. Xia, H. B. Wu, Y. Yan, X. W. (. D.). Lou, and X. Wang, "Ultrathin and ultralong single-crystal platinum nanowire assemblies with highly stable electrocatalytic activity," Journal of the American Chemical Society, vol. 135, no. 25, pp. 94809485, 2013.

[17] P. Song, X. Cui, Q. Shao, Y. Feng, X. Zhu, and X. Huang, "Networked Pt-Sn nanowires as efficient catalysts for alcohol electrooxidation," Journal of Materials Chemistry A, vol. 5, no. 47, pp. 24626-24630, 2017.

[18] W. Hong, J. Wang, and E. Wang, "Facile synthesis of PtCu nanowires with enhanced electrocatalytic activity," Nano Research, vol. 8, no. 7, pp. 2308-2316, 2015.

[19] S. Liu, Q. Zhang, Y. Li et al., "Five-fold twinned Pd2NiAg nanocrystals with increased surface $\mathrm{Ni}$ site availability to improve oxygen reduction activity," Journal of the American Chemical Society, vol. 137, no. 8, pp. 2820-2823, 2015.

[20] L. Ma, C. Wang, B. Y. Xia et al., "Platinum multicubes prepared by $\mathrm{Ni}^{2+}$-mediated shape evolution exhibit high electrocatalytic activity for oxygen reduction," Angewandte Chemie International Edition, vol. 54, no. 19, pp. 5666-5671, 2015.

[21] J. Liu, K. He, W. Wu, T. B. Song, and M. G. Kanatzidis, "In situ synthesis of highly dispersed and ultrafine metal nanoparticles from chalcogels," Journal of the American Chemical Society, vol. 139, no. 8, pp. 2900-2903, 2017.

[22] E. V. Shevchenko, D. V. Talapin, H. Schnablegger et al., "Study of nucleation and growth in the organometallic synthesis of magnetic alloy nanocrystals: the role of nucleation rate in size control of CoPt3 nanocrystals," Journal of the American Chemical Society, vol. 125, no. 30, pp. 9090-9101, 2003.

[23] M. Gong, G. Fu, Y. Chen, Y. Tang, and T. Lu, "Autocatalysis and selective oxidative etching induced synthesis of platinum-copper bimetallic alloy nanodendrites electrocatalysts," ACS Applied Materials \& Interfaces, vol. 6, no. 10, pp. 7301-7308, 2014.

[24] F. Q. Shao, J. J. Feng, Z. Z. Yang, S. S. Chen, J. Yuan, and A. J. Wang, "Cytosine assisted aqueous synthesis of AgPt hollow alloyed nanostructures as highly active electrocatalyst for ethylene glycol oxidation and hydrogen evolution," International Journal of Hydrogen Energy, vol. 42, no. 39, pp. 24767-24775, 2017.

[25] N. Sui, K. Wang, X. Shan et al., "Facile synthesis of hollow dendritic $\mathrm{Ag} / \mathrm{Pt}$ alloy nanoparticles for enhanced methanol oxidation efficiency," Dalton Transactions, vol. 46, no. 44, pp. 15541-15548, 2017.

[26] H.-H. Li, S.-Y. Ma, Q.-Q. Fu, X.-J. Liu, L. Wu, and S.-H. Yu, "Scalable bromide-triggered synthesis of Pd@ Pt core-shell ultrathin nanowires with enhanced electrocatalytic performance toward oxygen reduction reaction," Journal of the American Chemical Society, vol. 137, no. 24, pp. 7862-7868, 2015.

[27] M. Chen, B. Wu, J. Yang, and N. Zheng, "Small adsorbateassisted shape control of Pd and Pt nanocrystals," Advanced Materials, vol. 24, no. 7, pp. 862-879, 2012.

[28] K. Zhang, D. Bin, B. Yang, C. Wang, F. Ren, and Y. du, "Ruassisted synthesis of $\mathrm{Pd} / \mathrm{Ru}$ nanodendrites with high activity for ethanol electrooxidation," Nanoscale, vol. 7, no. 29, pp. 12445-12451, 2015.

[29] J. A. Michel, W. H. Morris Iii, and C. M. Lukehart, "Synthesis of shaped Pt nanoparticles using common anions or small molecules as shape-directing agents: observation of a strong halide or pseudo-halide effect," Journal of Materials Chemistry A, vol. 3, no. 5, pp. 2012-2018, 2015.
[30] H. Zhang, M. Jin, J. Wang et al., "Synthesis of Pd-Pt bimetallic nanocrystals with a concave structure through a bromideinduced galvanic replacement reaction," Journal of the American Chemical Society, vol. 133, no. 15, pp. 6078-6089, 2011.

[31] H. Wang, L. Wang, T. Sato et al., "Synthesis of mesoporous Pt films with tunable pore sizes from aqueous surfactant solutions," Chemistry of Materials, vol. 24, no. 9, pp. 1591-1598, 2012.

[32] W. Yu, W. Tu, and H. Liu, "Synthesis of nanoscale platinum colloids by microwave dielectric heating," Langmuir, vol. 15, no. 1, pp. 6-9, 1999.

[33] K. Eid, H. Wang, P. He et al., "One-step synthesis of porous bimetallic PtCu nanocrystals with high electrocatalytic activity for methanol oxidation reaction," Nanoscale, vol. 7, no. 40, pp. 16860-16866, 2015.

[34] W. Yu, M. Liu, H. Liu, X. Ma, and Z. Liu, "Preparation, characterization, and catalytic properties of polymer-stabilized ruthenium colloids," Journal of Colloid and Interface Science, vol. 208, no. 2, pp. 439-444, 1998.

[35] X. Yu, D. Wang, Q. Peng, and Y. Li, "Pt-M ( $\mathrm{M}=\mathrm{Cu}, \mathrm{Co}, \mathrm{Ni}, \mathrm{Fe})$ Nanocrystals: From Small Nanoparticles to Wormlike Nanowires by Oriented Attachment," Chemistry - A European Journal, vol. 19, no. 1, pp. 233-239, 2013.

[36] D. Zhao, J. Zhou, and N. Liu, "Characterization of the structure and catalytic activity of copper modified palygorskite/ $/ \mathrm{TiO}_{2}\left(\mathrm{Cu}^{2+}-\mathrm{PG} / \mathrm{TiO}_{2}\right)$ catalysts," Materials Science and Engineering: A, vol. 431, no. 1-2, pp. 256-262, 2006.

[37] Z. Dasdelen, Y. Yildiz, S. Eris, and F. Sen, "Enhanced electrocatalytic activity and durability of Pt nanoparticles decorated on GO-PVP hybride material for methanol oxidation reaction," Applied Catalysis B: Environmental, vol. 219, pp. 511-516, 2017.

[38] B. Hammer and J. K. Nørskov, "Theoretical surface science and catalysis-calculations and concepts," in Advances in Catalysis, Ed., vol. 45, pp. 71-129, Elsevier Academic Press Inc, San Diego, CA, USA, 2000. 


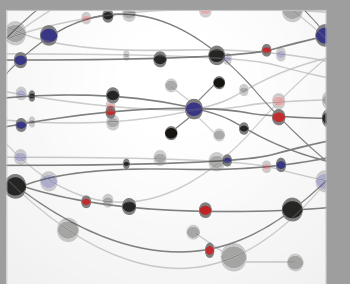

The Scientific World Journal
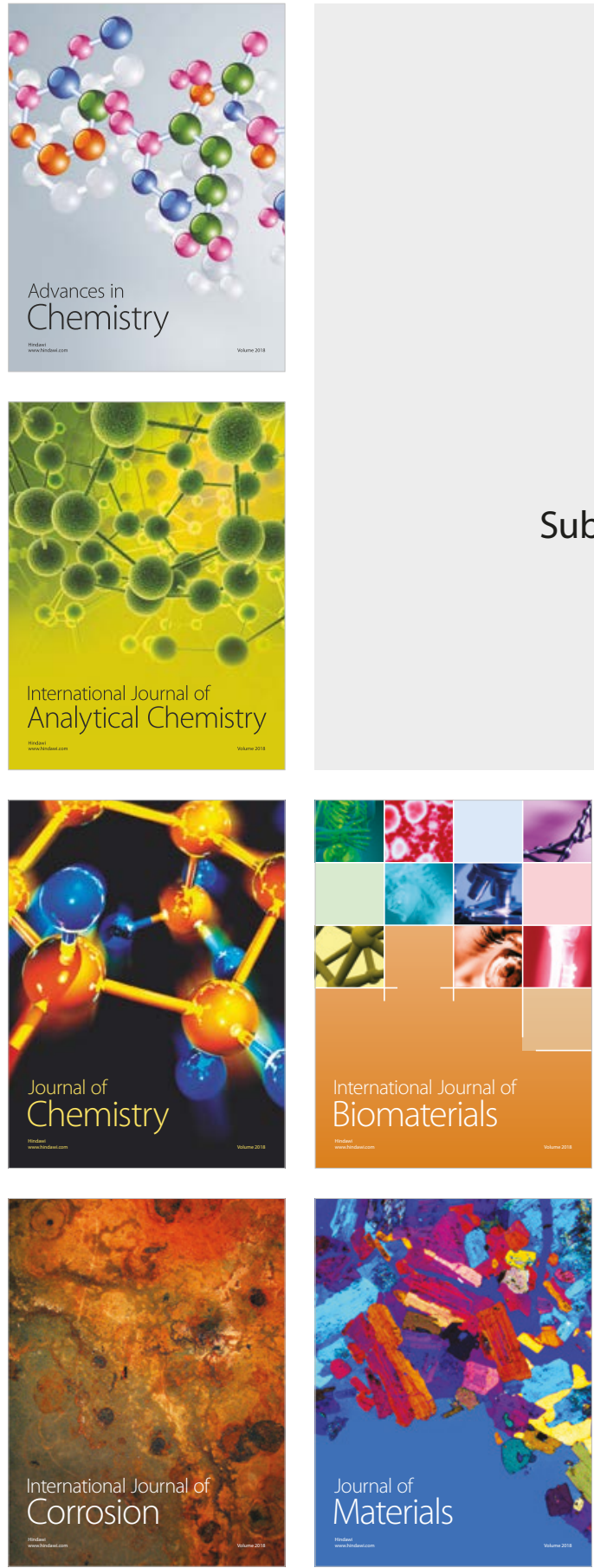

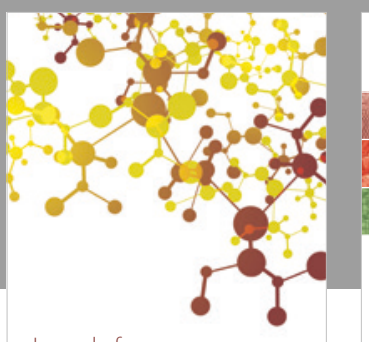

Journal of

Applied Chemistry
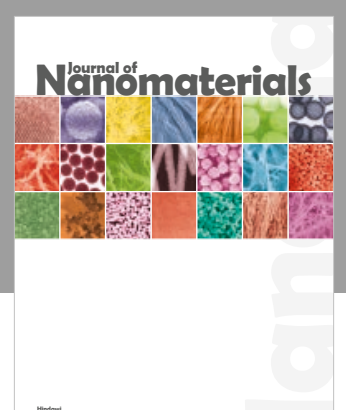

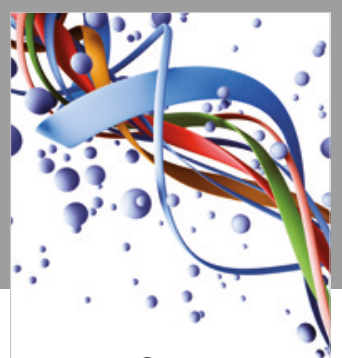

Scientifica

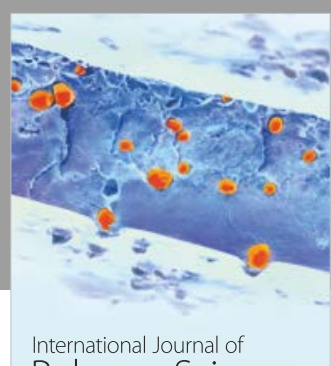

Polymer Science

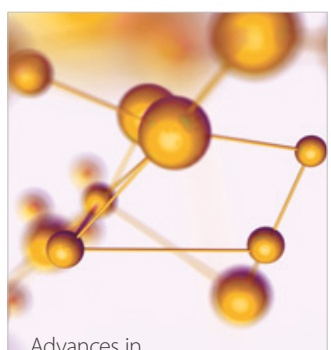

Physical Chemistry
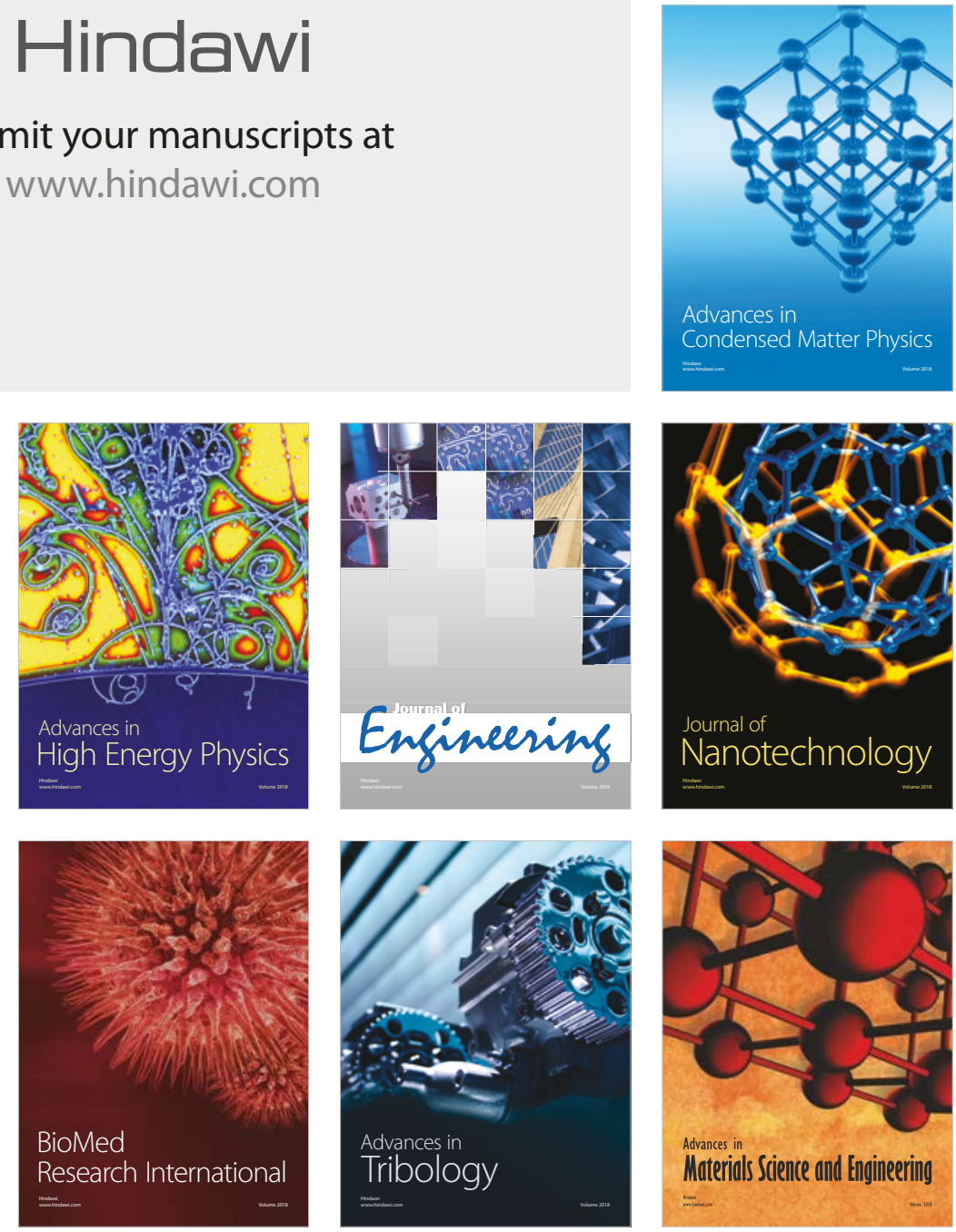